\title{
Unusual Histological Variant of a Primary Osseous Tumor of the Jaw in a 27-Year-Old Female: Review of the Literature and Case Presentation
}

\author{
Erisa Kola ${ }^{1 *}$, Leart Berdica ${ }^{1,2}$, Teona Bushati ${ }^{1,2}$, Alfred Aga ${ }^{3}$, Ali Guy ${ }^{4}$, \\ Ina Kola ${ }^{5}$, Edlira Horjeti ${ }^{6}$, Klejda Hoxha ${ }^{7}$, Fareeha Nasir ${ }^{8}$ and Juna \\ Musa $^{9}$ \\ ${ }^{1}$ Department of Pathology and Forensic Medicine, Faculty of Medicine, UMT, Tirane, \\ Albania \\ ${ }^{2}$ Department of Pathology, American Hospital, Tirane, Albania \\ ${ }^{3}$ Department of Head and Neck surgery, American Hospital, Tirana, Albania \\ ${ }^{4}$ Department of Physical Medicine and Rehabilitation, New York University, School of \\ Medicine-NYU, New York, USA \\ ${ }^{5}$ Department of Plastic Surgery and Burns, Mother Teresa Hospital Center, Tirane, \\ Albania \\ ${ }^{6}$ Family Doctor, Department of Family Medicine, Tirane, Albania \\ ${ }^{7}$ Medical Doctor, Family Medicine. Tirane, Albania \\ ${ }^{8}$ Intern, Department of Surgery, Christian Hospital Quetta, Pakistan \\ ${ }^{9}$ Postdoctoral Research Fellow, Department of Robotic Surgery, Mayo Clinic, Minnesota \\ *Corresponding Author: Erisa Kola, Department of Pathology and Forensic \\ Medicine, Faculty of Medicine, UMT, Tirane, Albania.
}

\begin{abstract}
Introduction: Osteosarcoma is the most common primary malignant bone tumor and represents $20 \%$ of head and neck malignancies. Meanwhile osteosarcoma of the jaw is a rare entity, distinct from the conventional type commonly arising in the long bones. According to literature it comprises $6.5-7 \%$ of all osteosarcomas. WHO has acknowledged several variants of osteosarcoma that differ in location, clinical manifestation and the degree of cytologic atypia [1]. These lesions can arise de novo and present as primary bone tumors, or in a pre-excisting condition such as previous head and neck radiation. Also, hereditary retinoblastoma, Paget's disease of bone, previous history of fibrous dysplasia, or trauma has been associated with the development of osteosarcomas. From a histopathologic point of view, osteosarcomas are commonly classified as osteoblastic, chondroblastic, or fibroblastic, although several unusual microscopic subtypes have also been reported.

Material and Methods: The aim of this article is to highlight the importance of clinical, radiological and histopathologic correlations in order to reach an accurate definite diagnosis in the setting of effective surgical management of this kind of lesions. We share our experience presenting a case of chondroblastic variant of osteosarcoma of the maxilla in a 27-year-old woman, and a critical review of the literature.

Conclusion: Osteosarcoma of the jaw is challenging both to diagnose and manage. The biological behavior of osteosarcomas in the jaws differs from tumors of other skeletal bones. Histopathological examination with adequate specimen evaluation is the key to accurate diagnosis and staging. Microscopically, the essential criterion of osteosarcomas is direct production of osteoid or immature bone by tumor cells. Osteosarcoma should always be considered in the differential diagnosis of expansile lesions of the jaws. Hence, early diagnosis and adequate surgical resection are the keys to better survival.

Keywords: Osteosarcoma; Chondroblastic Osteosarcoma; Osteosarcoma of Maxilla; Osseous Tumors
\end{abstract}

\section{Introduction}

Osteosarcoma is an aggressive primary bone tumor composed of connective tissue cells producing osteoid and bone. Although rare, head and neck malignant mesenchymal tumors (sarcomas) are a heterogeneous group of tumors with variable clinical presentations, pathologic classifications, and biologic behaviors. Osteosarcoma and rhabdomyosarcoma are the most common types of sarcomas in the craniofacial region. Osteosarcomas primarily affect the mandible and less frequently the maxilla, and the mean age for diagnosis is 30 years with heterogeneous sex distribution [3,4]. Osteosarcomas are classified as primary (central or surface) and secondary osteosarcomas arising in preexisting conditions. The etiology of primary osseous tumors is unknown. However, some risk factors have been attributed such as rapid bone growth as the incidence increases during adolescent growth and because of the typical location of tumor near the metaphyseal growth plate of the long bones. Contrarily, osteosarcoma of the jaw develops after adolescence which excludes rapid bone growth as the major etiologic 
factor. Other risk factors associated include environmental factors such as ionizing radiation and radioactive scanning agents, fibrous dysplasia, bone infarcts, chronic osteomyelitis, trauma and Paget's disease. Whereas various types of osteosarcomas have been defined, chondroblastic osteosarcoma is the most common variant of osteosarcoma of jaw bones. Generally, the signs and symptoms of osteosarcomas in the oral region are discreet and include regional swelling, low-intensity pain, paresthesia, changes in tooth position, loose teeth, and changes in the fit of prosthesis. They are often nonspecific and account for the considerable delay before the correct diagnosis is made.

\section{Case Presentation}

A 27-year-old female patient was presented to the department of Oral and Maxillofacial surgery with the chief complaint of a rapidly growing swelling in the right upper back tooth region associated with mild pain. She has been noticing the swelling over a period of 2 months. Her past medical history is irrelevant.

Clinical examination revealed an expansile swelling in the right maxillary region, near the alveolary process measuring $3.5 \mathrm{~cm} \times 2$ $\mathrm{cm}$. The swelling was tender on palpation and smooth in texture. Patient had parasthesia and pain in the involved areas. However, mouth opening was normal.

Panoramic radiograph showed an ill-defined radiolucent lesion in the right maxillary region involving the upper right alveolar process. Regional pathological lymph nodes were not detected.

Surgical intervent was performed with subtotal right maxillary removal.
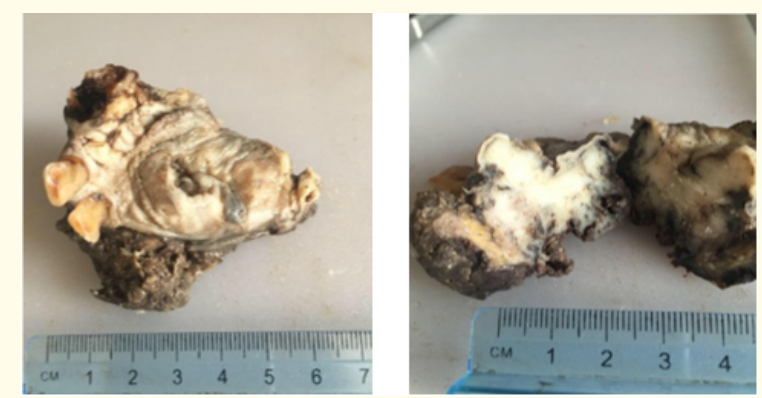

Figure 1: Macroscopic examination revealed an irregular firm white-gray lesion with undefined borders measuring $3.5 \times 2.3 \mathrm{~cm}$ as shown in the pictures.

Microscopic examination in H\&E stain showed evidence of a malignant neoplasm of mesenchymal origin characterized by the presence of irregular bone trabeculae, diffuse osteoid production admixed with atypical chondroblasts. At the periphery of the lesion are noted pleomorphism fusiform cells.

The histopathological examination confirms the diagnosis of Chondroblastic osteosarcoma located in the right maxilla.

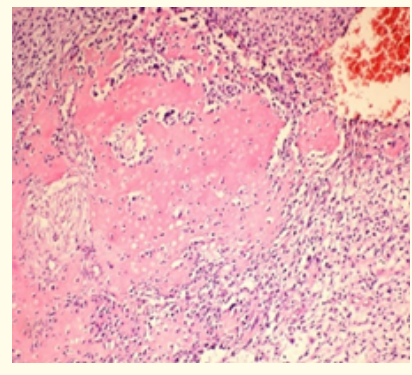

Figure 2: Low magnification of the lesion in H\&E stain revealing chondroid tissue with areas of osseous differentiation.

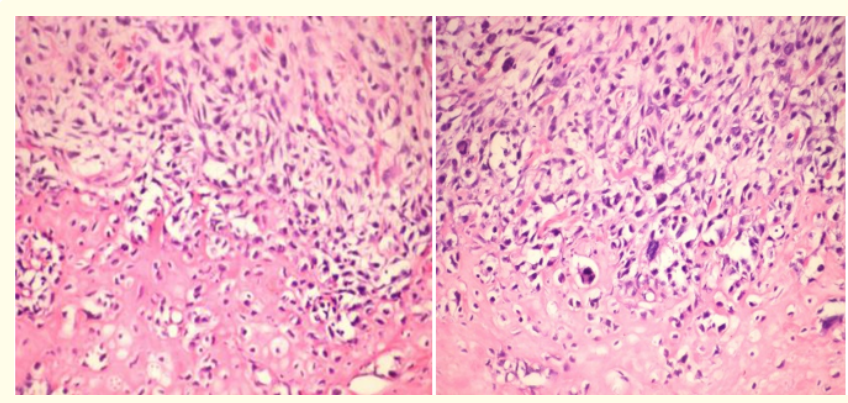

Figure 3a and 3b: Medium power view of the lesion, showing epithelioid and fusiform neoplastic chondrocytes.

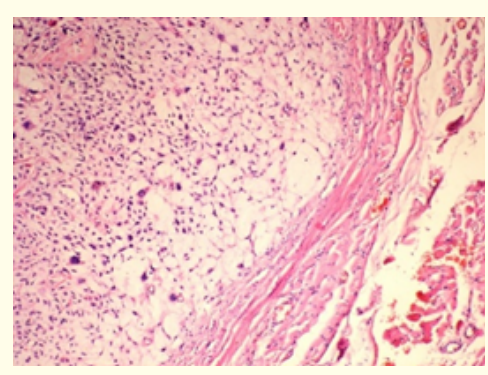

Figure 4: H\&E stain revealing areas of the lesion with high degree of pleomorphism and mitotic rate.

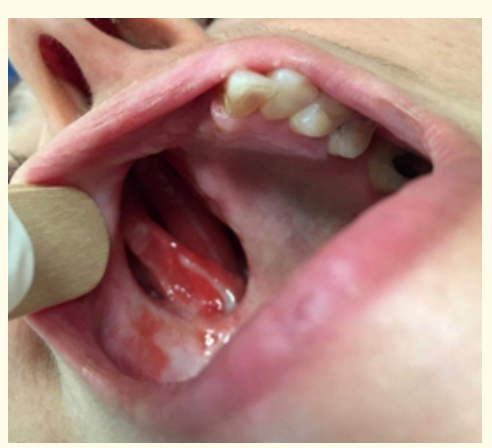

Figure 5: Post-operative clinical photo showing the lesion healed well after the surgery.

\section{Discussion}

The radiographic appearance of osteosarcoma depends on alternation of three factors: bone destruction, bone production with mineralization and periosteal new bone formation. Hence, lesions 
can appear radiolucent, radiolucent with fluffy cloud-like radio opaque areas or entirely radio opaque. Histologically the diagnosis of osteosarcoma is made when sarcomatous stroma involves osteoid or primitive bone [4]. The predominant histological variants described are osteoblastic, fibroblastic and chondroblastic variants and rarer subtypes such as epithelioid and telangiectatic have been described but they are not prognostically significant. According to WHO classification chondroblastic osteosarcoma is defined as a histological entity characterized by predominant presence of chondroid matrix.

Despite sharing common histopathological features, osteosarcoma of jaw bones and osteosarcoma of long bones are distinct biological entities. Osteosarcomas of the upper and lower jaws are challenging lesions in many ways. These tumors are very deforming, because of their anatomical region and affect the patient's quality of life. Thus, adequate surgical resection of these tumors is another challenge, especially of the maxilla, because of the complexity of the anatomy, and the resultant defect. However according to our literature data based on retrospective studies and case reports osteosarcoma of the maxilla presents a better prognosis than osteosarcoma of the long bones [5,10-13].

Some authors suggest that tumor size, location, and histologic grade are the main prognostic factors [7], with others affirming that tumor size does not appear to be of prognostic value [8]. The likelihood of cure in osteosarcomas of the jaws has been estimated at $60 \%$ to $70 \%$ [9], however early diagnosis and adequate surgical resection with control of local spread are essential for survival [14-24].

\section{Conclusion}

Osteosarcoma of the maxilla is a challenging clinical entity. Hence, chondroblastic osteosarcoma is a histopathological diagnosis. The management of all variants of osteosarcoma, including lowgrade, intramedullary, and juxtacortical osteosarcomas, are identical with complete surgical excision of the tumor together with wide safe surgical margins. Although other treatment modalities such as chemotherapy and radiation therapy have been proposed, the majority of the tumors are clinically identified in advanced stages and require combination treatment. Since loco-regional recurrence is the main cause of mortality in osteosarcomas, resection with negative margin is the gold standard of the treatment.

This case report highlights the importance of continuous oral examinations of both soft and hard oral tissues in patients under orthodontic treatment. Early diagnosis has a great impact on treatment planning and prognosis of the patients.

\section{Bibliography}

1. Fletcher CDM., et al. "World Health Organization, classification of tumors: Pathology and genetics of tumors of soft tissue and bone". Lyon: IARC Press (2013).

2. Murphey MD., et al. "The many faces of osteosarcoma”. Radiographic 17 (1997): 1205-1231.
3. Bennett JH., et al. "Osteosarcoma of the jaws: A 30-year retrospective review". Oral Surgery, Oral Medicine, Oral Pathology, and Oral Radiology 90 (2000): 323-333.

4. Doval DC., et al. "Osteosarcoma of the jaw bones". British Journal of Oral and Maxillofacial Surgery 35.5 (1997): 357- 362.

5. Martin RG., et al. "Primary bone tumors.in: Cancer patient care at M.D Anderson Hospital and Tumor Institute". Year Book Medical, Chicago (1976): 485-495.

6. Caron AS., et al. "Osteogenic sarcoma of the facial and cranial bones. A review of forty-three cases". The American Journal of Surgery 122 (1971): 719-725.

7. Granados-Garcia M., et al. "Free osseous and soft tissue surgical margins as prognostic factors in mandibular osteosarcoma". Oral Oncology 42 (2006): 172-176.

8. Altuwairgi O., et al. "Maxillary chondroblastic sarcoma: presentation of two cases and a literature review". Journal of Oral and Maxillofacial Surgery 54 (1996): 1357-1364.

9. Mendenhall WM., et al. "Head and neck osteosarcoma". American Journal of Otolaryngology 32 (2011): 597-600.

10. Hewitt KM., et al. "Parosteal osteosarcoma: case report and review of the literature". Head and Neck 30 (2008): 122-126.

11. Mardinger 0., et al. "Osteosarcoma of the jaw. The Chaşm Sheba Medical Center experience". Oral Surgery, Oral Medicine, Oral Pathology, and Oral Radiology 91 (2001): 445-451.

12. Garrington GE., et al. "Osteosarcoma of the jaws: analysis of 56 cases". Cancer 20 (1967): 337-391.

13. Finklestein JB. "Osteosarcoma of the jaw bones". Radiologic Clinics of North America 8 (1970): 425-433.

14. Nissanka EH., et al. "Clinicopathological analysis of osteosarcoma of jaw bones". Oral Diseases 13 (2007): 82-87.

15. Kabani SP and Pollack RP. "Osteosarcoma presenting as supracrestal bone formation". Journal of Periodontology 65 (1994): 93-96.

16. Raymond AK., et al. "Osteosarcoma of head and neck". Laboratory Investigation 60 (1989): 76.

17. Patterson A., et al. "Periosteal osteosarcoma of the maxilla: a case report and review of literature". Journal of Oral and Maxillofacial Surgery 48 (1990): 522-526.

18. Khammissa RA., et al. "Osteosarcoma of the jaw: A brief review and a case report". South African Dental Journal 64 (2009): 220-221.

19. Rosenthal MA., et al. "High-grade maxillofacial osteosarcoma: Evolving strategies for a curable cancer". Oral Oncology 39 (2003): 402-404. 
20. Seidl RO., et al. "Mesenchymal chondrosarcomas of the facial skull”. HNO 49 (2001): 744-749.

21. Junior AT., et al. "Clinicopathological and immunohistochemical analysis of twenty-five head and neck osteosarcomas". Oral Oncology 39 (2003): 521-530.

22. ALQahtani D., et al. "Epithelioid Osteosarcoma of the Maxilla: A Case Report and Review of the Literature". International Journal of Surgical Pathology 23 (2015): 495-499.

23. Al-Yahya A., et al. "Maxillary Osteosarcoma: Two Case Reports and Literature Review". Oral Health and Dental Management 13 (2014): 525-528.

24. Yildiz FR., et al. "Gnathic osteosarcomas, experience of four institutions from Turkey". International Journal of Clinical and Experimental Pathology 7 (2014): 2800-2808.

\section{Assets from publication with us}

- Prompt Acknowledgement after receiving the article

- Thorough Double blinded peer review

- Rapid Publication

- Issue of Publication Certificate

- High visibility of your Published work

Website: https://www.actascientific.com/

Submit Article: https://www.actascientific.com/submission.php Email us: editor@actascientific.com

Contact us: +919182824667 